\title{
Realidade do envelhecimento populacional frente às doenças crônicas não transmissíveis
}

\author{
Reality of population ageing in front of chronic diseases non-transmissible \\ Realidad del envejecimiento poblacional frente las enfermedades crónicas no \\ transmisibles
}

Isabela Simieli*, Letícia Aparecida Resende Padilha, Cristiane Fernandes de Freitas Tavares.

\section{RESUMO}

Objetivo: Analisar a realidade da população senil, as características socioeconômicas, a prevalência de doenças crônicas e os fatores de riscos associados. Métodos: Trata-se de um estudo observacional transversal, cujas informações foram adquiridas por um questionário aplicado a 204 idosos, em duas Unidades Básicas de Saúde em Franca-SP e por pesquisa de dados em prontuários. O questionário continha dados sobre identificação, condições socioeconômicas, fatores de risco, doenças crônicas e a história familiar. Resultados: Dos 204 entrevistados 68,13\% eram mulheres; 54,41\% brancos; 45,58\% casados; $78,43 \%$ vivendo com algum familiar; $87,25 \%$ inativos; $62,22 \%$ estudaram apenas até a $4^{\text {a }}$ série; $66,17 \%$ com renda menor que dois salários mínimos. Foi relatado o sedentarismo em $64,22 \%$ deles, com IMC $\geq 25 \mathrm{~kg} / \mathrm{m}^{2} \mathrm{em} 43,14 \%$. As patologias mais comuns foram a hipertensão atingindo $77,71 \%$ e a osteoartrose em $54,9 \%$ deles. Conclusão: Com a análise, concluímos que as mulheres buscam mais o cuidado a saúde, as quais estão expostas a hipertensão como uma das doenças crônicas não transmissíveis, sendo um dos fatores de risco correlacionados a doenças cardiovasculares. Assim, torna-se possível a realização de ações de prevenção e promoção de saúde, a fim de minimizar as complicações por meio do diagnóstico precoce, tratamento e seguimento adequado.

Palavras-chave: Doença Crônica, Idosos, Prevenção de Doenças.

\begin{abstract}
Objective: To analyze the reality of the senile population, the socioeconomic characteristics, the prevalence of chronic diseases and the associated risk factors. Methods: This was a cross-sectional observational study, whose information was acquired by a questionnaire applied to 204 elderly people, in two Basic Health Units in Franca-SP and by data search in medical records. The questionnaire contained data on identification, socioeconomic conditions, risk factors, chronic diseases and family history. Results: Of the 204 interviewed, $68.13 \%$ were women; $54.41 \%$ white; $45.58 \%$ married; $78.43 \%$ living with a relative; $87.25 \%$ inactive; $62.22 \%$ studied only until grade $4 ; 66.17 \%$ with income lower than two minimum wages. Sedentary lifestyle was reported in $64.22 \%$ of them, with $\mathrm{BMI} \geq 25 \mathrm{~kg} / \mathrm{m}^{2}$ in $43.14 \%$. The most common pathologies were hypertension reaching $77.71 \%$ and osteoarthrosis in $54.9 \%$. Conclusion: With the analysis, we conclude that women seek more health care, which are exposed to hypertension as one of the chronic noncommunicable diseases, being one of the risk factors correlated to cardiovascular diseases. Thus, it is possible to carry out prevention and health promotion actions in order to minimize complications through early diagnosis, treatment and adequate follow-up.
\end{abstract}

Key words: Chronic Disease, Aged, Desease Prevention.

1Universidade de Franca (UNIFRAN), Franca- São Paulo. *E-mail: cristiane.tavares@unifran.edu.br 


\section{RESUMEN}

Objetivo: Analizar la realidad de la población senil, las características socioeconómicas, la prevalencia de enfermedades crónicas y los factores de riesgo asociados. Métodos: Este fue um estudio observacional transversal, cuya información se obtuvo mediante um cuestionario aplicado a 204 ancianos, en dos Unidades de Salud Básica em Franca-SP y mediante la búsqueda de datosen registros médicos. El cuestionario contenía datos sobre identificación, condiciones socioeconómicas, factores de riesgo, enfermedades crónicas y antecedentes familiares. Resultados: De los 204 entrevistados, $68.13 \%$ fueronmujeres; $54.41 \%$ blancos; $45.58 \%$ casados; $78.43 \%$ vivendo com un familiar; $87,25 \%$ inactivos; $62.22 \%$ estudió hasta el grado $4 ; 66.17 \%$ com um ingreso inferior a dos salarios mínimos. Se informo

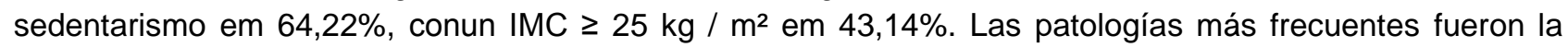
hipertensión arterial que alcanzó $77,71 \%$ y la artroses $54,9 \%$. Conclusión: con el análisis, concluímos que las mujeres buscan más atención médica, que están expuestas a la hipertensión como una de las enfermedades crónicas no transmisibles, siendo uno de los factores de riesgo relacionados con las enfermedades cardiovasculares. Entonces, es posible realizar acciones de prevención y promoción de la salud para minimizar las complicaciones a través del diagnóstico temprano, tratamento y seguimento adecuado.

Palabras clave: Enfermedad crónica, Ancianos, Prevención de Enfermedades.

\section{INTRODUÇÃO}

O envelhecimento populacional iniciou-se no final do século XIX na Europa Ocidental e ampliou-se, nas últimas décadas, por vários países do Terceiro Mundo, incluindo o Brasil (CARVALHO JAM e GARCIA RA, 2003). Neste, observou-se, a partir da década de 60 - cuja taxa de natalidade era de 5,8 filhos por mulher, uma acelerada queda neste índice em um período de 30 anos, reduzindo-se em $60 \%$, cerca de 2,5 filhos por mulher (CARVALHO JAM e GARCIA RA, 2003; MOREIRA MM, 1998). Associado à diminuição da mortalidade e ao aumento da expectativa de vida, contribuíram para o processo de envelhecimento populacional (CARVALHO JAM e GARCIA RA, 2003; RAMOS LR, et al, 1987).O número de senis ( $\geq 60$ anos de idade) aumentou de 3 milhões em 1960, para 7 milhões em 1975 e 14 milhões em 2002 e estudos estimam que alcançará 32 milhões em 2020 (COSTA MFL e VERAS R, 2003).

Segundo a Organização Mundial da Saúde (OMS), define-se idoso aquele com 60 anos ou mais, em países em desenvolvimento e com 65 anos ou mais em países desenvolvidos (INAGAKI RK, et al, 2008). O envelhecimento está associado ao processo biológico de declínio que ocorre com o tempo, e é próprio daquelas pessoas que atingiram ao estado final do ciclo vital, no qual a idade define como limitativo ao bemestar biológico, psicológico e comportamental (MOREIRA MM, 1998). O envelhecimento é um processo multifatorial e subjetivo, não sendo relevante apenas a idade: destaca-se a saúde e qualidade de vida, 0 que requer uma compreensão mais abrangente como aspectos demográficos, psicossociais e econômicos (DIAS AM, 2007).

Os fenômenos associados ao rápido envelhecimento têm impacto socioeconômico e demográfico, em particular aqueles que resultam da redução da taxa de crescimento populacional e das mudanças na estrutura etária, ampliando, assim, o contingente de idosos e produzindo demandas sociais que requerem respostas políticas adequadas (KUCHEMANN BA, 2012; RAMOS LR, et al, 1987).

O envelhecimento é um dos maiores desafios da saúde pública atual uma vez que enfermidades próprias ganham maior expressão e consequentemente existe uma maior demanda por serviços de saúde. Além disso, as internações hospitalares são mais frequentes nesta faixa etária, o tempo de ocupação de leitos é maior e a maioria das doenças são crônicas, o que exige medicações, exames periódicos e cuidados contínuos (COSTA MFL e VERAS R, 2003).

As doenças crônicas não transmissíveis (DCNTs) são doenças multifatoriais com impacto global, que cursam com elevado número de mortes, incapacidades e perda de qualidade de vida, além de causar 
impacto econômico nas famílias e na sociedade. (Organization WH, 2011). Caracteriza-se por um grupo de doenças marcadas pela ausência de microrganismos no modelo epidemiológico, pela nãotransmissibilidade, pelo longo curso clínico e pela irreversibilidade (LESSA I, 1998).

Indubitavelmente, atinge todas as classes socioeconômicas, principalmente os idosos com baixa renda e escolaridade, por serem justamente os mais expostos aos fatores de risco e com menor acesso aos conhecimentos e aos serviços de saúde (BONITA R, et al, 2013). A maioria das doenças crônicas é causada por combinações de fatores sociais, culturais, ambientais e comportamentais (MCQUEEN DV, 2007).

Os números crescentes de DCNTs refletem efeitos negativos da globalização, urbanização, alimentação demasiadamente calórica, sedentarismo e vícios (Organization WH, 2011). Os fatores de risco podem ser analisados pelo estilo de vida do paciente, que inclui medidas tais como prática de atividade física, hábitos alimentares, ou seja, comportamentos adquiridos, estando, portanto, parcialmente sob seu controle, já que o ambiente social e a situação socioeconômica são fatores que interferem nas escolhas individuais (MALTA DC, et al, 2015).

Dentre as condições de risco destacam-se as comportamentais, como tabagismo, etilismo, ingestão de alimentos pouco nutritivos e sedentarismo (MALTA DC, et al, 2012). O fumo é responsável por quase $10 \%$ dos casos de doenças cardiovasculares. O sedentarismo aumenta em $20 \%$ a $30 \%$ o risco de mortalidade. Estima-se que, entre os óbitos causados por álcool, mais de $50 \%$ sejam devido às DCNTs. (DUNCAN BB, 2012). Os padrões alimentares adotados podem ser prejudiciais, como exemplo, o consumo excessivo de sal que aumenta o risco de doenças cardiovasculares, assim como o alto consumo de carne processada e de ácidos graxos, que também aumenta esses riscos, além de causar diabetes.

No Brasil, em 2013, as DCNTs foram a causa de aproximadamente $72,6 \%$ das mortes, culminando na elaboração do "Plano de Ações Estratégicas para o Enfrentamento das Doenças Crônicas Não Transmissíveis no Brasil, 2011-2022", elaborado pelo Ministério da Saúde com participação de diversas outras instituições relevantes. As propostas estão estruturadas em três eixos: vigilância, informação, avaliação e monitoramento; promoção da saúde e cuidado integral (MALTA DC e SILVA JB, 2013; MALTA DC, et al, 2015). Este visa estabelecer metas e compromissos, ou seja, definir ações e investimentos necessários para os desafios das DCNTs e seus fatores de risco, nos próximos dez anos (MALTA DC e SILVA JB, 2013; Saúde M, 2011).

Diante desse cenário e segundo as projeções as quais indicam um crescimento no número de idosos no Brasil, há a necessidade de estudos objetivando avaliar a realidade da saúde desses indivíduos frente às DCNTs e fatores de riscos relacionados a elas, para que os cuidados da saúde acompanhem as mudanças na pirâmide etária do país e para que a nova perspectiva possa ajudar a programar políticas de saúde local, medidas preventivas e intervenções baseadas nessa população.

\section{MÉTODOS}

Após a aprovação do projeto pelo Comitê de Ética em Pesquisa da Universidade de Franca, foi realizado um estudo observacional transversal, o qual as informações foram adquiridas em um único momento através de entrevista (com aplicação de questionário) e análise de prontuários de 204 pacientes idosos. Foram abordados pacientes $\geq 60$ anos, de ambos os sexos, cadastrados em duas Unidades Básicas de Saúde da cidade de Franca - SP.

No questionário aplicado na sala de espera das unidades, foi feito a identificação do paciente (nome, idade, sexo, estado civil, etnia) e o levantamento dos fatores socioeconômicos (escolaridade, trabalho e renda per capta em salários mínimos da família), dos fatores de risco para DCNTs (dieta, sedentarismo, excesso de peso - Índice de Massa Corporal > $25 \mathrm{~kg} / \mathrm{m} 2$, uso de tabaco e álcool) e das DCNTs existentes.

Através da análise dos prontuários dos pacientes, foram avaliados dados referentes às doenças e medicamentos em uso para confirmação das mesmas. Nas análises estatísticas foi utilizado o programa GraphPad, Software Incorporated, Prism, versão 6.0. 


\section{RESULTADOS}

Na pesquisa realizada, quanto aos fatores socioeconômicos e étnicos, observou-se a preponderância do sexo feminino, a predominância de brancos e casados. Em relação à moradia, a maior parte relatou viver com algum familiar. Quanto à ocupação, a maioria mencionou não exercer nenhuma atividade laboral. No que diz respeito à escolaridade, o relato mais comum foi ter cursado apenas o ensino fundamental e quanto à renda, a maioria dos participantes informou receber menos de dois salários mínimos (Tabela 1).

Tabela 1 - Porcentagem dos dados étnicos e socioeconômicos.

\begin{tabular}{|c|c|c|c|c|}
\hline & & Quantidade & Porcentagem & Total \\
\hline \multirow[t]{2}{*}{ Sexo } & Feminino & 139 & $68,13 \%$ & 204 \\
\hline & Masculino & 65 & $31,86 \%$ & \\
\hline \multirow[t]{5}{*}{ Etnia } & Brancos & 111 & $54,41 \%$ & 204 \\
\hline & Pardos & 54 & $26,47 \%$ & \\
\hline & Negros & 32 & $15,68 \%$ & \\
\hline & Amarelos & 5 & $2,45 \%$ & \\
\hline & Indígenas & 2 & $0,98 \%$ & \\
\hline \multirow[t]{4}{*}{ Estado Civil } & Casados & 93 & $45,58 \%$ & 204 \\
\hline & Viúvos & 63 & $30,88 \%$ & \\
\hline & Divorciados & 30 & $14,70 \%$ & \\
\hline & Solteiros & 18 & $8,82 \%$ & \\
\hline \multirow[t]{2}{*}{ Moradia } & Moram com Familiares & 160 & $78,43 \%$ & 204 \\
\hline & Sozinhos & 44 & $21,57 \%$ & \\
\hline \multirow[t]{2}{*}{ Ocupação } & Não trabalham & 178 & $87,25 \%$ & 204 \\
\hline & Trabalham & 26 & $12,75 \%$ & \\
\hline \multirow[t]{9}{*}{ Escolaridade } & Nunca Estudou & 24 & $11,76 \%$ & 204 \\
\hline & $1^{\mathrm{a}}$ a $4^{\mathrm{a}}$ série & 112 & $62,22 \%$ & \\
\hline & $5^{\mathrm{a}}$ a $8^{\mathrm{a}}$ série & 34 & $18,88 \%$ & \\
\hline & Ensino médio completo & 20 & $11,11 \%$ & \\
\hline & Superior completo & 9 & $5,00 \%$ & \\
\hline & Ensino médio incompleto & 3 & $1,66 \%$ & \\
\hline & Superior incompleto & 1 & $0,55 \%$ & \\
\hline & Pós-graduação & 1 & $0,55 \%$ & \\
\hline & <2 salários mínimos & 135 & $66,17 \%$ & 201 \\
\hline Fatores & Entre 2 e 5 salários mínimos & 61 & $29,90 \%$ & \\
\hline Socieconômicos & > 5 salários mínimos & 5 & $2,45 \%$ & \\
\hline
\end{tabular}

Fonte: Simieli I, Padilha LAR, Tavares CFF, 2018.

Foi observado que 189 dos 204 (92.65\%) dos participantes do estudo apresentam pelo menos um dos fatores de riscos gerais para desenvolvimento de DCNT. Entre todos os idosos, os fatores de risco analisados, os mais prevalentes foram o sedentarismo relatado por $131(64,21 \%)$ idosos, $88(43,14 \%)$ com 
IMC ( $\left.\geq 25 \mathrm{~kg} / \mathrm{m}^{2}\right), 58(28.43 \%)$ idosos com dieta rica em gorduras, $43(21,07 \%)$ idosos com dieta pobre em frutas, verduras e legumes, $36(17,65 \%)$ idosos fazerem uso frequente de álcool e $28(13,73 \%)$ o uso de tabaco (Gráfico 1).

Gráfico 1 - Distribuição dos participantes aos fatores de riscos para o desenvolvimento de DCNT.

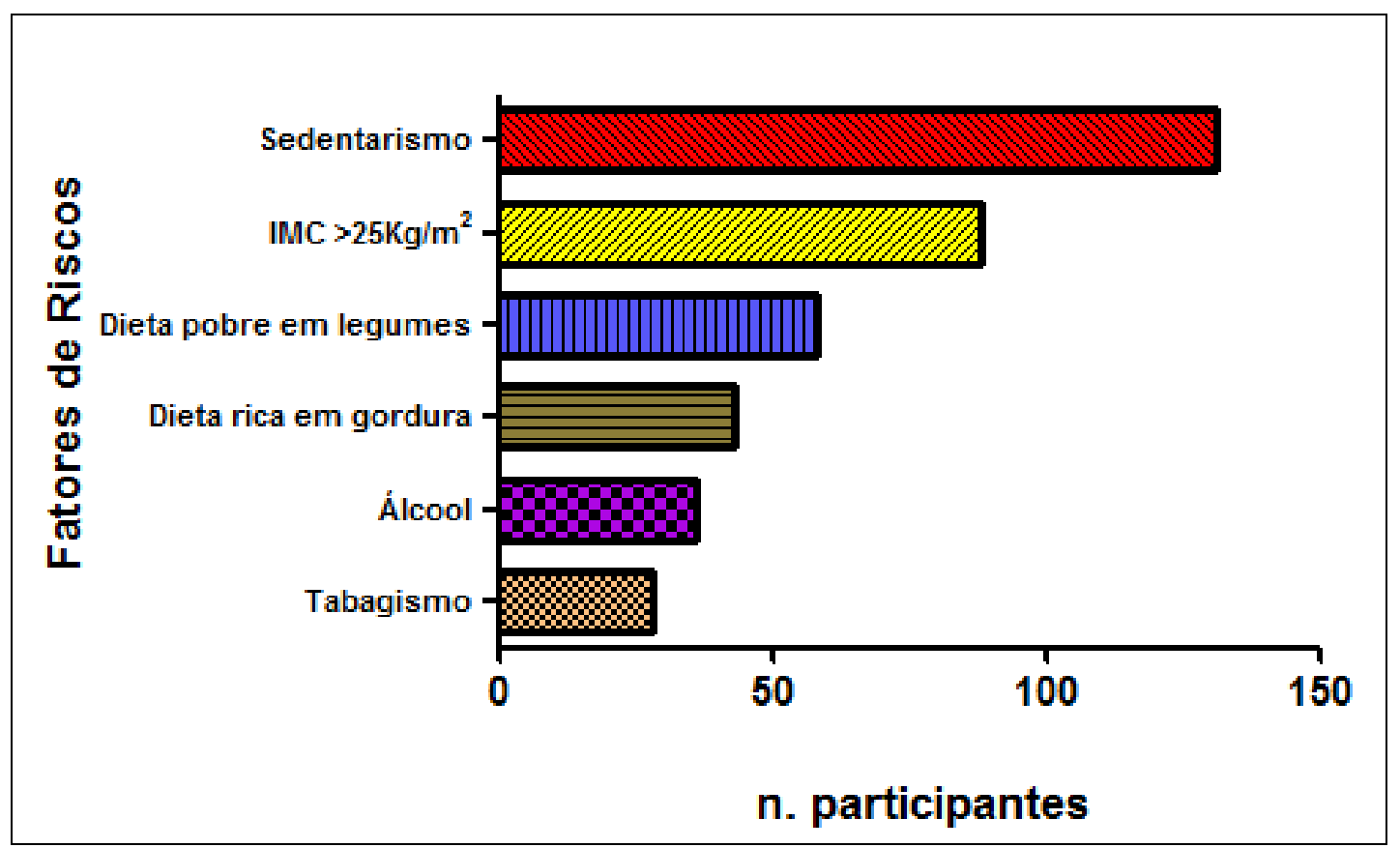

Fonte: Simieli I, Padilha LAR, Tavares CFF, 2018.

$\mathrm{Na}$ abordagem de doenças crônicas existentes, 184 idosos apresentam pelo menos uma doença crônica, enquanto somente 20 participantes são hígidos. Em meio as diversas doenças crônicas, foram observadas $143(77,71 \%)$ idosos com hipertensão arterial sistêmica, $70(38,04 \%)$ com osteoartrose, 68 $(36,95 \%)$ com diabetes, $43(23,36 \%)$ com depressão, 38 (20,65\%) com osteoporose, $24(13,04 \%)$ com dislipidemia, $14(7,6 \%)$ apresentaram ansiedade, $9(4,89 \%)$ anemia das doenças crônicas, $8(4,34 \%)$ um tipo de neoplasia, $6(3,26 \%)$ doença pulmonar crônica, 6 (3,26\%) com doença musculoesquelética, 3 $(1,63 \%)$ com Parkinson e $3(1,63 \%)$ com Alzheimer (Gráfico 2).

Gráfico 2 - Distribuição das DCNT nos participantes.

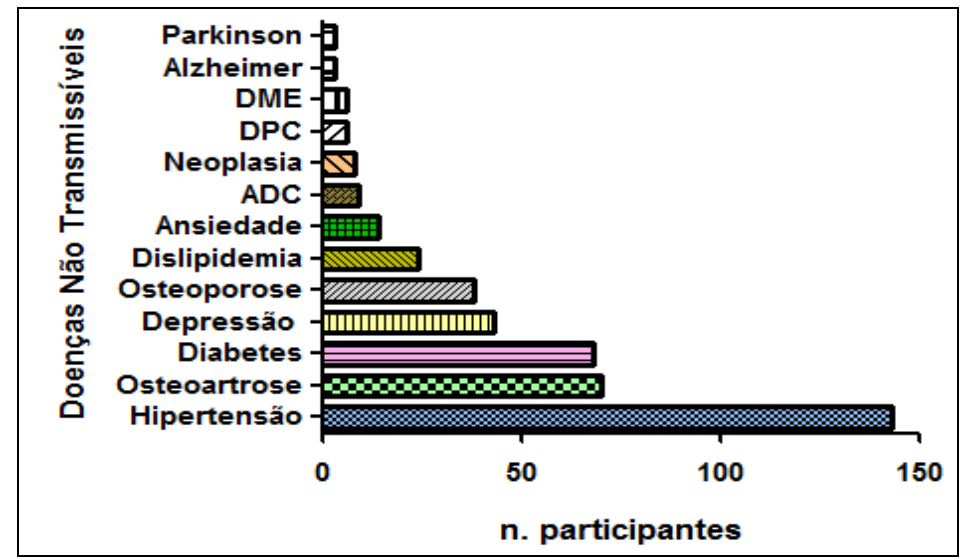

Legenda: DME: doença muscular esquelética, DPC: doença pulmonar crônica, ADC: anemia das doenças crônicas. Fonte: Simieli I, Padilha LAR, Tavares CFF, 2018. 
Perante análise dos resultados do IMC, levando em consideração a tabela da OMS, $43(21,07 \%)$ dos idosos apresentaram IMC adequado $\left(18,5 \leq \mathrm{IMC}<25 \mathrm{~kg} / \mathrm{m}^{2}\right)$. Em contrapartida, 93 (45,58\%) dos idosos se encontraram acima do peso adequado em relação a altura e $68(33,33 \%)$ participantes não souberam informar. Dos participantes acima do peso, $48(51,61 \%)$ deles exibiram sobrepeso $\left(25 \leq \mathrm{IMC}<29.9 \mathrm{~kg} / \mathrm{m}^{2}\right)$, $36(38,7 \%)$ obesidade grau $1\left(30 \leq \mathrm{IMC}<34,9 \mathrm{~kg} / \mathrm{m}^{2}\right)$ e $9(9,67 \%)$ obesidade grau $2(35 \leq \mathrm{IMC}<39.9$ $\left.\mathrm{kg} / \mathrm{m}^{2}\right)$.

\section{DISCUSSÃO}

Definir o que se considera uma população idosa é complexo, pois delimitar um início exato é improvável - envolve fatores sociais, econômicos e culturais. Esse processo por ser marcado tanto por aparência física, surgimento de comorbidades crônico-degenerativas, redução da capacidade física/mental ou pela perda de papéis sociais, quanto como pela idade (CAMARANO AA e KANSO S, 2013). No Brasil, os idosos são aqueles com idade $\geq 60$ anos, e ainda dentro desse grupo, existem os "muito idosos", aqueles com mais de 80 anos. (MACHADO L e PERNIN E, 2010). Dentro dessa divisão, observou-se que 187 dos participantes são idosos e 17 dos entrevistados são considerados como muitos idosos. Com o envelhecimento, diminuise a incidência e prevalência de doenças infectocontagiosas, porém elevam-se as taxas de adoecimento por DCNTs.

O patriarcalismo estabeleceu a cultura de que o homem é marcado pela invulnerabilidade, força e virilidade. Estes percebem o cuidado à saúde como algo não peculiar, pois demonstrar medo e insegurança coloca em risco à masculinidade (CAVALCANTI JRD, et al, 2014; GOMES R, et al, 2007). Dessa forma, ignoram a importância da prevenção de doenças. Observou-se que $68,13 \%$ dos entrevistados eram mulheres, enquanto apenas $31,86 \%$ eram do sexo masculino. Esse resultado também pode ser explicado pelo aumento da expectativa de vida feminina, consequência de fatores biológicos e de uma reduzida exposição a fatores de risco, como violência e acidentes. A luta pela igualdade de gênero, ao incluir as mulheres ao mercado de trabalho, torna-as mais independentes, agindo como um fator protetor à saúde visto a satisfação proporcionada. Contudo, a maior longevidade feminina acarreta períodos mais longos de exposição às doenças degenerativas, que pode ocasionar redução da qualidade de vida destas (CAMARANO AA e KANSO S, 2013).

Em relação à raça, observa-se que a expectativa de vida é menor onde os cidadãos negros são maioria, fato observado comparando-se países pobres, tais como alguns africanos - nos quais a maioria são negros e a expectativa de vida é baixa, com as nações desenvolvidas, onde a maioria são brancos e com índice de envelhecimento elevado (BARROS CA e BRANCOS SID, 2017). No Brasil, os negros enfrentam piores situações trabalhistas, reduzidos salários e ínfimo acesso aos serviços de saúde, tornando-os mais susceptíveis às doenças crônicas (MALTA DC, et al, 2015). Foi observada uma minoria de cor negra no estudo, representando $15,68 \%$ da amostra em comparação ao número de brancos, $54,31 \%$, e também uma maior prevalência de comorbidades, já que dos 32 negros entrevistados 31 tinham alguma patologia, sendo a mais incidente a hipertensão arterial. $O$ número de idosos negros com renda inferior a 2 salários corresponde a 21 do total da amostra, sendo que somente 9 possuem a renda entre 2 e 5 salários mínimos e apenas 2 maior que 5 salários.

No tocante à escolaridade, os resultados apontaram um elevado número de idosos com baixos níveis, predominando entre as séries mais básicas, uma vez que a maioria dos participantes possuía apenas o antigo primário, ou seja, de 1aㅡ a $4^{\text {a }}$ série (JUNIOR EGS, et al, 2014). Essas estatísticas refletem a cultura passada, visto que até a década de 50 a alfabetização era pouco valorizada. Essa situação foi se modificando após o processo de industrialização, sendo que atualmente quem não tem acesso à linguagem e escrita se torna marginalizado e privado de informações. (FILHO PPS, et al, 2014).

O aumento da longevidade alterou os modelos familiares, já que muitos idosos possuem elevado grau de dependência familiar, seja por questões de saúde ou financeira. Há diferentes cenários, sendo os mais comuns o idoso que vive sozinho e aqueles que vivem com parentes, principalmente os filhos (GOMES R, et al, 2007). 
Foi observado que $78,43 \%$ viviam com algum familiar, sendo que apenas $21,57 \%$ moravam sozinhos. Contudo, muitas destas famílias não estão preparadas para serem cuidadores, sobretudo se o idoso possuir alguma comorbidade crônica. Quando surgem situações de dependência, a família necessita passar por um processo de reorganização de suas estruturas para lidarem com o processo de fragilidade (PAVARINI SCI, et al, 2009).

Apesar da maioria ainda morar com os familiares, é crescente a proporção de idosos vivendo sozinhos, possivelmente devido aos avanços nos meios de comunicação e a melhor qualidade na saúde. Esta situação pode ser vista principalmente entre os muitos idosos e mais em mulheres, principalmente associado ao fato de muitas serem viúvas e pelo crescente número de divórcios (CAMARANO AA e KANSO S, 2013). Foi observado que $45,09 \%$ eram casados, $30,88 \%$ viúvos, $14,70 \%$ divorciados e apenas $8,82 \%$ solteiros. Dados revelam que a mulher, quando viúva, prefere viver sozinha enquanto os homens preferem reconstruir sua família por meio de uma nova união, e quando não há sucesso nisso, preferem morar com familiares a morar só, visto possuírem uma maior dependência para ajudar nos seus cuidados (MELO NV, et al, 2009). Em corroboração a isso, foi observado no estudo que $63,07 \%$ dos homens eram casados.

Em nosso estudo foi observado que $87,25 \%$ dos idosos não executavam nenhum tipo de trabalho. Com o envelhecimento, encerra-se o ciclo produtivo de sua vida e começa uma nova fase, muitas vezes cujo dinheiro é insuficiente para suas necessidades. Dessa forma, o idoso pode sentir que não pertence mais a sociedade, ficando desamparado, o que culmina em um fator de risco para o desenvolvimento de patologias, principalmente a depressão (MELO NV, et al, 2009). A renda, apesar de estimular os idosos a buscarem independência optando por morarem sozinhos, também pode fazer com que a família fique em situação de interesse, dependentes do idoso (PAULO MA, et al, 2013). O mais observado foram os idosos dependentes da família, visto que a maioria recebia menos que 2 salários mínimos, uma das principais queixas, citadas pelos idosos - o dinheiro insuficiente para suas necessidades, principalmente devido aos elevados gastos com a saúde.

A expressão "fator de risco" refere-se a uma terminologia de relevância no campo das DCNTs, podendo estes serem modificáveis - tais como sedentarismo, alcoolismo, tabagismo, excesso de peso (IMC $\geq 25$ $\mathrm{kg} / \mathrm{m}^{2}$ ), dieta rica em gorduras e pobre em frutas verduras e legumes; e não modificáveis - idade, sexo, raça, estado civil, escolaridade, ocupação, moradia e renda (CASADO L, 2009). Estas afecções caracterizam-se por causas multifatoriais e refletem o reconhecimento de mecanismos etiológicos e fisiopatológicos que podem levar ao aparecimento de uma doença (REGO RA, et al, 1990). Nota-se que 189 idosos participantes (92.65\%) apresentaram pelo menos um dos fatores de riscos gerais. O conhecimento da prevalência destes fatores, em especial os de natureza comportamental, é essencial por serem sinais de alerta para o crescimento da morbimortalidade associada a essas doenças (CASADO L, 2009).

A modernização modificou os padrões comportamentais da sociedade: trouxe uma maior oferta de alimentos, mas houve diminuição no gasto de energia (DUARTE BM, 2010). $\mathrm{Na}$ amostra, o fator de risco mais prevalente foi sedentarismo com 64,22\% (131 idosos), sendo avaliado como um dos principais agravantes para enfermidades cardiovasculares e, neste contexto, foi notório a relação entre estilo de vida sedentário e hipertensão arterial sistêmica, sendo que 92 dos 131 idosos sedentários são hipertensos. (MARTINS LCG, et al, 2015). Além disso, está associada a cerca de 10 a 16\% dos cânceres de mama, cólon e reto e diabetes, sendo responsável por 1,9 milhões de morte no mundo (DUARTE BM, 2010).

Segundo a OMS, o consumo insuficiente de frutas, legumes e verduras (consumo diário inferior a 400 gramas ou, aproximadamente, cinco porções por pessoa) é responsável anualmente por 2,7 milhões de mortes e por $31 \%$ das doenças isquêmicas do coração, $11 \%$ das doenças cerebrovasculares e $19 \%$ dos cânceres gastrointestinais acontecidos em todo o mundo (MALTA DC, et al, 2011). O presente estudo verificou baixo consumo desses alimentos, 43 idosos (21.08\%), e elevada frequência de consumo de gorduras saturadas, 58 idosos (28.43\%), corroborando, deste modo, com os dados da Pesquisa de Orçamentos Familiares 2008-2009, que exibiu aspectos negativos sobre o padrão alimentar dos brasileiros, incluindo alto aporte de gorduras não saudáveis e baixo consumo de frutas, legumes e verduras (NASCIMENTO LS, et al, 2015).

REAS/EJCH | Vol.Sup.37 | e1511 | DOI: https://doi.org/10.25248/reas.e1511.2019 Página 7 de 9 
Uma das formas mais utilizadas para avaliação da prevalência do excesso de peso e obesidade é o Índice de Massa Corporal (IMC). Considera-se com excesso de peso aqueles que apresentarem IMC maior que $25 \mathrm{Kg} / \mathrm{m}^{2}$ e com obesidade os que apresentarem IMC maior que $30 \mathrm{Kg} / \mathrm{m}^{2}$ (GIGANTE DP, et al, 2009). Foi notório que 88 dos idosos (43.14\%) apresentavam IMC $\geq 25 \mathrm{~kg} / \mathrm{m}^{2}$.

O tabagismo é avaliado pela OMS como a principal causa de morte evitável e um dos principais fatores de risco para DCNTs (MALTA DC, et al, 2011). Na maioria dos países existe uma correlação entre tabagismo, baixa renda e baixa escolaridade, sendo que no Brasil indivíduos com baixa escolaridade têm uma probabilidade cerca cinco vezes maior de serem fumantes do que aqueles que têm o terceiro grau (DUARTE BM, 2010). Neste contexto, 28 idosos do estudo afirmam ser tabagistas. Destes, 21 (75\%) possuíam renda menor que 2 salários mínimos e $17(60,71 \%)$ participantes tabagistas estudaram da $1^{\text {a a }} 4^{\text {a }}$ série (antigo primário).

As DCNTs são uma das principais causas de óbito, tendo seus fatores de risco evitáveis e passíveis de intervenção. São responsáveis por $72 \%$ das mortes (DUARTE BM, 2010; Saúde M, 2013). Na análise do estudo das DCNTs existentes, 184 idosos apresentam pelo menos uma doença crônica, enquanto somente 20 participantes eram hígidos. Entre os resultados, as enfermidades mais comuns faziam referência a hipertensão arterial sistêmica, osteoartrose e diabetes. Este dado corrobora a favor da diretriz das doenças crônicas, uma vez que traz a informação de que entre as doenças, as de causas cardiovasculares constituem a grande maioria delas, sendo a hipertensão arterial sistêmica (HAS) a mais prevalente, aumentando progressivamente com a idade (MINISTÉRIO DA SAÚDE, 2013).

\section{CONCLUSÃO}

Dentre os dados analisados, concluímos que as mulheres buscam mais o cuidado a saúde, as quais estão expostas a hipertensão como uma das DCNTs, sendo um dos fatores de risco correlacionados a doenças cardiovasculares. Assim, torna-se essencial o reconhecimento dos fatores que levam a população a desenvolver essas doenças e a análise das patologias mais prevalentes. Identificando-se o perfil de exposição populacional e o seu estilo de vida, torna-se possível executar ações de promoção da saúde e prevenção primária, controlando os fatores de risco ou por meio de ações que minimizem complicações tais como diagnóstico precoce, tratamento e controles adequados.

\section{REFERÊNCIAS}

1. CARVALHO JAM, GARCIA RA. O envelhecimento da população brasileira: um enfoque demográfico. Cad.SaúdePública. 2003; 19(3): 725-33.

2. MOREIRA MM. O envelhecimento da população brasileira: intensidade, feminização e dependência. Revista brasileira de estudos de população. 1998; 15(1): 78-93.

3. RAMOS LR, et al. Envelhecimento populacional: uma realidade brasileira. Rev. Saúde Públ. 1987; 21(3): $211-24$.

4. COSTA MFL, VERAS R. Saúde pública e envelhecimento. Cad. Saúde Pública. 2003; 19(3): 700-1.

5. INAGAKI RK, et al. A vivência de uma idosa cuidadora de um idoso doente crônico. 2008; 17(2): 232-40.

6. DIAS AM. O processo de envelhecimento humano e a saúde do idoso nas práticas curriculares do curso de fisioterapia da UNIVALI campus Itajaí: um estudo de caso. Dissertação (Mestrado em Saúde e Gestão do Trabalho). Universidade do Vale do Itajai, Vale do Itajaí, 2007; 189 p.

7. KUCHEMANN BA. Envelhecimento populacional, cuidado e cidadania: velhos dilemas e novos desafios. Soc. Estado. 2012; 27(1): 165-80.

8. World Health Organization. Global status report on noncommunicable diseases 2010. Geneva: World Health Organization. 2011; 176.

9. LESSA I. Introdução à epidemiologia das doenças cardiovasculares no Brasil. In: O adulto brasileiro e as doenças da modernidade: epidemiologia das doenças crônicas não-transmissíveis. São Paulo: HUCITEC; Rio de Janeiro: ABRASCO, 1998. p. 73-76.

10. BONITA R, et al. Country action country actions to meet UN commitments on non-communicable diseases: a stepwise approach. Lancet. 2013; 381 (9866): 575-84.

11. MCQUEEN DV. Continuing efforts in global chronic disease prevention. Prev Chronic Dis. 2007; 4(2): 1-2. 
12. MINISTÉRIO DA SAÚDE (BR). Secretaria de Vigilância em Saúde. Departamento de Análise de Situação de Saúde. Plano de ações estratégicas para o enfrentamento das doenças crônicas não transmissíveis (DCNT) no Brasil 2011-2022. Brasília: Ministério da Saúde; 2011.

13. MALTA DC, et al. Estilos de vida da população brasileira: resultados da Pesquisa Nacional de Saúde, 2013. Epidemiol. Serv. Saúde, Brasília, 24(2): 217-226, abr-jun 2015.

14. MALTA DC, et al. Resultados do monitoramento dos Fatores de risco e Proteção para Doenças Crônicas Não Transmissíveis nas capitais brasileiras por inquérito telefônico, 2008. Ver Bras Epidemiol 2012; 15(3): 639-50.

15. DUNCAN BB. Doenças Crônicas Não Transmissíveis no Brasil: prioridade para enfrentamento e investigação. Rev SaúdePública 2012; 46 (Supl): 126-34.

16. MALTA DC, SILVA JB. O Plano de Ações Estratégicas para o Enfrentamento das Doenças Crônicas Não Transmissíveis no Brasil e a definição das metas globais para o enfrentamento dessas doenças até 2025: uma revisão. Epidemiol Serv. Saude 2013; 22 (1): 151-64.

17. CAMARANO AA, KANSO S. Envelhecimento da população brasileira: uma contribuição demográfica. In: Freitas EV, PY L. Tratado de Geriatria e Gerontologia. 3.ed. Rio de Janeiro: Guanabara Koogan; 2013. P. 133-52.

18. MACHADO L, PERNIN E. Atenção à Saúde da Pessoa Idosa e Envelhecimento. Série Pactos pela Saúde. 2010; $12: 1-46$.

19. GOMES R, et al. Por que os homens buscam menos os serviços de saúde do que as mulheres? As explicações de homens com baixa escolaridade e homens com ensino superior. Cad. SaúdePública. 2007, mar; 23(3): 565-74.

20. CAVALCANTI JRD, et al. Assistência Integral a Saúde do Homem: necessidades, obstáculos e estratégias de enfrentamento. Esc Anna Nery Rev de Enferm. 201, Out-Dez; 18(4): 628-34.

21. BARROS CA, BRANCOS SID. Envelhecimento da população negra, desigualdade racial e qualidade de vida. Rev. Tema UNIESP. 2017.

22. MALTA DC, et al. Diferenciais dos fatores de risco de Doenças Crônicas não Transmissíveis na perspectiva de raça/cor. Rev. Ciência \& Saúde Coletiva. 2015; 20(3): 713-25.

23. JUNIOR EGS, et al. Influência da escolaridade no desempenho cognitivo de idosos. In: Anais do 1ํㅡㄹ Congresso Internacional de Educação e Inclusão, 2014; Campina Grande- PB. Editora Realize.

24. FILHO PPS, et al. Escolarização e seus efeitos no letramento de idosos acima de 65 anos. Rev. Bras. Geriatr. Gerontol. 2014; 17(3): 589-600.

25. PAVARINI SCI, et al. Família e Vulnerabilidade social: um estudo com octogenários. Rev Latino-am Enfermagem. 2009 maio-junho; 17(3).

26. MELO NV, et al. Arranjo domiciliar de idosos no Brasil: análises a partir da Pesquisa Nacional por Amostra de Domicílios (2009). Rev. Bras. Geriatr. Gerontol. 2016; 19(1): 139-151.

27. MENDES MRSSB, et al. A situação social do idoso no Brasil: uma breve consideração. Acta Paul Enferm. 2005; 18(4): 422-6.

28. PAULO MA, et al. A relação entre renda e composição domiciliar dos idosos no Brasil: um estudo sobre o impacto do recebimento do Benefício de Prestação Continuada. R. bras. Est. Pop. 2013; 30: 525-43.

29. CASADO L. Fatores de Risco para Doenças Crônicas não Transmissíveis no Brasil: uma Revisão Sistemática. Rev Bras de Cancerologia. 2009; 55(4): 379-88.

30. REGO RA, et al. Fatores de risco para doenças crônicas não-transmissíveis: inquérito domiciliar no município de São Paulo, SP (Brasil). Metodologia e resultadospreliminares. Rev Saúde Pública. 1990; 24 (4): 277-85.

31. DUARTE BM. Fatores de risco e proteção para doenças crônicas não transmissíveis na população de Belo Horizonte. Dissertação (Mestrado em Saúde e Enfermagem). Escola de Enfermagem da UFMG, Belo Horizonte, 2010; $107 \mathrm{p}$.

32. MALTA DC, et al. Fatores de risco e proteção para doenças crônicas não transmissíveis entre beneficiários da saúde suplementar: resultados do inquérito telefônico Vigitel, Brasil, 2008. Ver Ciência \& Saúde Coletiva. 2011; 16(3): 2011-22.

33. MARTINS LCG, et al. Sedentary lifestyle in individuals with hypertension. Rev Bras Enferm. 2015; 68(6): 697-704.

34. NASCIMENTO LS, et al. Fatores de risco para doenças crônicas não transmissíveis e variáveis sociodemográficas de servidores públicos. Rev Bras Promoç Saúde. 2015 abr/jun; 28(2): 230-39.

35. GIGANTE DP, et al. Prevalência de excesso de peso e obesidade e fatores associados, Brasil, 2006. Ver Saúde Pública. 2009; 43(2): 83-89.

36. MINISTÉRIO DA SAÚDE. Diretrizes para o cuidado das pessoas com doenças crônicas nas redes de atenção à saúde e nas linhas de cuidado prioritárias. 2013. 1 ed: 1-30. 